\title{
Polarization independent high transmission large numerical aperture laser beam focusing and deflection by dielectric Huygens' metasurfaces
}

\author{
Aytekin Özdemir ${ }^{a, b *}$, Zeki Hayran ${ }^{\mathrm{b}}$, Yuzuru Takashima ${ }^{\mathrm{a}}$, Hamza Kurt \\ ${ }^{a}$ College of Optical Sciences, University of Arizona, 1630 E. University Blvd., Tucson, AZ 85721, USA \\ ${ }^{b}$ Department of Electrical and Electronics Engineering, TOBB University of Economics and Technology, Ankara 06560,Turkey
}

\begin{abstract}
In this letter, we propose all-dielectric Huygens' metasurface structures to construct high numerical aperture flat lenses and beam deflecting devices. The designed metasurface consists of two-dimensional array of all-dielectric nanodisk resonators with spatially varying radii, thereby introducing judiciously designed phase shift to the propagating light. Owing to the overlap of Mie-type magnetic and electric resonances, high transmission was achieved with rigorous design analysis. The designed flat lenses have numerical aperture value of 0.85 and transmission values around $80 \%$. It also offers easy fabrication and compatibility with available semiconductor technology. This spectrally and physically scalable, versatile design could implement efficient wavefront manipulation or beam shaping for high power laser beams, as well as various optical microscopy applications without requiring plasmonic structures that are susceptible to ohmic loss of metals and sensitive to the polarization of light.
\end{abstract}

Keywords: Metasurfaces, Metamaterials, Phase shift, Subwavelength structures

\section{Introduction}

Recently, metamaterials and metasurfaces have attracted significant attention in the optics community $[1,2]$. Metamaterials are artificial materials which are constituted by nanostructures, and optical resonant effects of these nanostructures allow metamaterials to have unique optical 
properties due to negative dielectric permittivity, $\varepsilon$ and magnetic permeability, $\mu$. Metasurfaces are two-dimensional (2D) counterparts of metamaterials with thicknesses much smaller than the wavelength of the incident light, enabling complete manipulation of the basic properties of light beams, such as phase, amplitude and polarization [3,4]. Compared to metamaterials, metasurfaces do not require complex fabrication methods and can be fabricated with single step lithographical techniques. Thus, they are well suited for mass production and can be integrated onto a photonic chip. In addition, metamaterials have high loss in the optical regime; this also limits their practical applications at this particular frequency regime [5]. Even if metasurfaces are ultra-thin, they still effectively manipulate the phase, amplitude, and polarization of light in transmission or reflection mode. Many planar analogs of the traditional bulky optical components, such as lenses [6-13], anti-reflection coatings [14], axicons [8,15], polarization converters [16], optical vortex generators [17], and absorbers [18] have been demonstrated by using metasurfaces.

Electromagnetic phase control is one of the simplest and probably one of the most unique applications of metasurfaces. Full $2 \pi$ phase control is the key for implementing various applications, such as beam steering, structured light generation, or lensing. Remarkably, metasurfaces can conveniently modulate the phase of electromagnetic waves in various ways, such as dynamical phase control, and geometrical phase control. Dynamical phase control relates to the change in the optical path to acquire the required optical path difference. Metasurfaces introduce interfacial phase discontinuities along the optical path as an alternative approach to realize very compact and flat dynamical phase elements. However, dynamical phase metasurfaces are circular polarization insensitive. The other types of metasurfaces, which are called geometrical phase metasurfaces, have also been proposed and demonstrated 
experimentally $[19,20]$. Geometrical phase metasurfaces utilize space-variant polarization manipulation of the incident field in order to induce local phase retardation. This type of phase retardation is actually a Pancharatnam-Berry (PB) phase shift which in some cases is an undesired effect but can be utilized for spin filter and beam shaping applications. By designing two-layer metasurfaces (one of them is polarization or spin-insensitive and the other one is polarization or spin-controlled), geometric phase and dynamic phase metasurface elements can be integrated to implement ultra-compact polarization beam-splitters and spin filters [21,22]. Majority of initial studies on metasurfaces focused on metal-dielectric structures, which have low efficiency due to nonradiative Ohmic losses at optical frequencies especially operating in transmission mode $[23,24]$. Many plasmonic-based metasurfaces and flat lenses were recently designed using V-shaped antennas and rotationally asymmetric nanostructures [8,25-27]. Such systems typically had energy efficiency less than $20 \%$ due to increased Ohmic losses in optical frequency regions. On the other hand, almost all metals melt at high temperatures and, thus, they are not well suited for high-power laser applications.

Due to the abovementioned drawbacks of metals, significant work has been done in developing dielectric analogs of metasurfaces in visible and near-infrared range, since many dielectric materials have very low absorption loss in these optical frequencies. Most of the previous designs relied on high contrast grating approaches with nano-pillar structures of higher aspect ratio [11]. High refractive index materials like silicon were studied to show strong Mie-type scattering mechanism [28]. The well-known "Huygens' principle" states that the new wavefront is determined by the sum of secondary wavelets generated by all points on the previous wavefront. Low-loss dielectric nanoresonators are designed to emulate the behavior of the forward-propagating elementary wavelets known from the Huygens' principle [3]. In other 
words, Huygens' sources can be achieved by using polarizable subwavelength particles that sustain both magnetic and electric dipolar resonances. The arrangement of many of such particles in a plane creates special reflectionless sheets or metasurfaces that are called Huygens' metasurfaces [29]. Silicon nanodisk structures were especially demonstrated to support the simultaneous excitations of electric and magnetic dipole resonances [30-34]. By varying the geometric parameters or the aspect ratio of silicon nanodisks, electric and magnetic dipole resonances can be overlapped at the same frequency. This also suppresses the backward scattering since electric and magnetic resonances will cancel each other in backscattering while interfering constructively in forward direction by realizing Kerker's condition [35]. Thus, these two dipole resonance mechanisms allow us to tailor the phase shift over $0-2 \pi$ while having a high transmission and almost zero reflection. Also, silicon is a common material used mostly in semiconductor industry, which may benefit from mature semiconductor fabrication technology. Due to CMOS compatibility and the relative ease of fabrication, silicon-based structures continue to be an important element of the recent state-of-the-art nanophotonic device studies [36]. As a result, it is advantageous to use these silicon nanodisk metasurface structures to implement high-power laser beam manipulation with low-reflection losses and polarizationindependence property.

In this paper, we report the design and analysis of an efficient, ultra-thin planar metasurface lens and beam deflecting device which operates at a wavelength of $\lambda=1064 \mathrm{~nm}$, by utilizing the recently developed Huygens' metasurfaces. Designed devices rely on silicon nanodisk structures which can support both Mie-type electric and magnetic dipole resonances to realize phase manipulation and completely suppress reflection losses. By locally changing the radius of the silicon nanodisk elements, abrupt phase changes were realized. A flat metasurface lens with a 
high numerical aperture value of 0.85 and with transmissivity as high as $80 \%$ and a metasurface beam deflecting device with beam deflecting angle of $6.9^{\circ}$ with transmissivity of $75 \%$ have been demonstrated. With robust optimization algorithms, the design proposed here has great potential to be improved as a future study. Our design offers excellent polarization insensitive operation due to high degree of symmetry. Also, much higher transmission than the previous studies with the same wavelength of operation at $1064 \mathrm{~nm}$ was achieved [37]. The proposed design is also easy to fabricate with standard single step electron beam lithography based conventional planar semiconductor fabrication methods due to ultrathin thickness and very small aspect ratio of the designed structures comparing to recent studies with longer pillars [7]. Based on these outstanding properties and spectral scalability, these devices can be utilized in applications where operation at distinct known wavelengths is needed such as high power laser beam systems and fluorescence microscopy techniques.

\section{Design and Results}

\subsection{Design of the unit cell}

The proposed Huygens' metasurfaces are designed using high index silicon nanodisks, which have subwavelength periodicity and are embedded in a host low refractive index silica material. Other comparative design forms such as silicon free-standing in silica substrate may also be considered. Note that the spectral widths of the Mie-type resonances are determined by the proper choice of the dielectric environment. The refractive-index contrast between the nanodisk and surrounding media significantly affects the mode confinement of the electric and magnetic

dipole resonances. Furthermore, by geometrical tuning of nanodisk dimensions, the spectral positions of the two resonances can be adjusted [30,32]. The schematic of 2D all-dielectric 
Huygens' metasurface lens is shown in Fig. 1(a) as an example. Silicon nanodisk array period $(\mathrm{P})$, nanodisk height $(\mathrm{H})$ and, nanodisk radius $(\mathrm{R})$ are the three basic structural and geometric design parameters that control the transmission amplitude and phase of the Huygens' metasurfaces as shown in Fig. 1(b).

The metasurfaces considered here are designed for a wavelength of $1064 \mathrm{~nm}$ and optimized for operation in transmission under normal incident transverse-electric (TE) polarized plane wave. Design is polarization insensitive due to the symmetry of the structural nanodisk elements. In the analysis, the refractive index of Silicon $(\mathrm{Si})$ and Silica $\left(\mathrm{SiO}_{2}\right)$ are 3.56 and 1.45 , respectively as they are extracted from Palik's handbook [38]. Also, the imaginary part of the refractive index of silicon was taken as about $8 \times 10^{-4}$ at $1064 \mathrm{~nm}$ from the data of Palik [38]. Using finitedifference time-domain (FDTD) method software, FDTD solutions from Lumerical Inc. the transmission and phase modulation of the nanodisk arrays with different geometric parameters are calculated. The nanodisks are assumed to be embedded in a host substrate of fused Silica (SiO2) in the simulations. The periods of the arrays are optimized in order to get a broader overlap region of the electric and magnetic dipole resonances in the spectrum and, thus, a broader transmission region with respect to variation of nanodisk geometric parameters. It is estimated that a lattice period value of $620 \mathrm{~nm}$ is the best option to design a high NA (numerical aperture) flat lens with a numerical aperture value around 0.85 due to the Nyquist sampling criterion $\left(P<\frac{\lambda}{2 N A}\right)$. Since periods of the arrays are fixed both in the $x$ and $y$ directions, and due to rotationally symmetric nanodisk elements, polarization independence is ensured. During unit cell analysis, the incident plane wave at $1064 \mathrm{~nm}$ is normal to the array plane with polarization along the $x$ direction, and periodic boundary conditions are adopted in each boundary of the unit cell to decrease the amount of computation time. 
Figs. 2(a) and 2(b) show the calculated contours of the transmissivity and transmission phase of the nanodisk arrays for different illumination wavelength and radii $(\mathrm{R})$. By varying the nanodisk radius, relatively high transmission and full phase coverage $(0-2 \pi)$ can be achieved at the same time for the nanodisk height of $170 \mathrm{~nm}$ (nanodisk embedded in $\mathrm{SiO}_{2}$ ), respectively. It is clear that this enables arbitrary wavefront control by precisely manipulating the spatial distribution of the radius variation of dielectric nanodisk resonators. A fixed lattice period of $620 \mathrm{~nm}$ both provides a broad area with high transmissivity and allows easy fabrication with high tolerance as well as less complex design. The more the lattice period reduces, the more coupling occurs between the adjacent unit cells and high transmission region significantly reduces.

Figs. 2(c) and 2(d) clearly show the transmission and the phase modulation for different nanodisk radii while the wavelength is fixed at $1064 \mathrm{~nm}$ as the operation wavelength. For convenience, here the phase modulation of $130 \mathrm{~nm}$ radius is set to be zero, and all negative phases are changed to positive by adding $2 \pi$ to them. This result serves as look-up data for choosing the proper disk radius for the phase manipulation required. In order to achieve any desired phase profile at each spatial location $(x, y)$, an appropriate nanodisk diameter is chosen to minimize the transmission error $\Delta T=\left|e^{i \varphi(x, y)}-T(D) e^{i \varphi(D)}\right|$, where $T(D) e^{i \varphi(D)}$ is the complex transmission coefficient. The other important point in Fig. 2(c) is that transmission goes down especially towards the larger radius values of the nanodisks. Considering the fact that periodic boundary conditions are used in the full-wave simulation and nanodisks have fixed period and height, coupling effect gets more pronounced with the larger nanodisk radii. Having radius as the only degrees of freedom makes the design and fabrication easier but results in a trade-off against transmission. Metasurfaces with high aspect ratio subwavelength building 
blocks can be considered, but fabrication may be harder due to tapering problem of the vertical side-walls of the fabricated structures, and more advanced fabrication methods are required [7].

Note that when the fabrication errors are taken into account, the phase and transmission values may departure from the ideal values. Rigorous optimization algorithms and sensitivity analyses need to be performed to further improve the performance and address the fabrication errors $[39,40]$. However, there is still no sufficient optimization study for especially all-dielectric high NA flat lenses due to time consuming full-wave simulation requirement. It should be also noted that chromatic dispersion here is not a significant problem since design is focused on the manipulation of laser beams, which are intrinsically narrowband.

\subsection{Design of $1 D$ beam deflector}

We firstly start with the design of a metasurface with beam steering ability, in order to show the fundamental ability of the all-dielectric Huygens' metasurfaces to manipulate the wavefront of a transmitted beam for various applications. By introducing in-plane phase gradient $d \varphi / d x$ at the interface between two media, new degrees of freedom to control light propagation are attained in metasurfaces. The desired phase function for the beam deflecting functionality is given by

$$
\varphi(x)=k_{x} x=\frac{2 \pi}{\lambda} n_{i} \sin \left(\theta_{t}\right) x
$$

where $k_{x}$ is a proportionality factor or wave vector along $x, \lambda$ is the wavelength of the incident beam in free-space, $n_{i}$ is the refractive index of the material at the incidence side, $\theta_{t}$ is the deflection angle. For normal incidence and $n_{i}$, the deflection angle $\theta_{t}$ given by [41]

$$
\theta_{t}=\sin ^{-1}\left(\frac{\lambda}{2 \pi \cdot n_{i}} \frac{d \varphi}{d x}\right)
$$


In our simulations, we design periodic supercells of total length $6.2 \mu \mathrm{m}$ as shown in Fig. 3(a), which consist of 10 silicon nanodisks with periodicity of $P=620 \mathrm{~nm}$. The radius of each silicon nanodisk is properly selected so that there is total $2 \pi$ phase difference throughout the super-cell

with width of $6.2 \mu \mathrm{m}$. Note that there is $\frac{2 \pi}{10}=36^{\circ}$ phase difference between two adjacent unit cells. Meanwhile, in the FDTD simulations, periodic boundary conditions are adopted along the $y$-axis, whereas perfectly matched layer boundary conditions are employed along the $x$-axis and $z$-axis in order to reduce the computational resources. The designed deflection angle is about $6.8^{\circ}$, and the simulated deflection angle $\theta$ is $6.9^{\circ}$ relative to the surface normal, which is close as shown in Fig. 3(c). The desired phase-difference profile of the metasurface beam deflector based on Eq. (1) and Eq. (2) is indicated by the dashed green line in Fig. 3(b). Red triangles are the phases of each corresponding silicon nanodisk for the beam deflector. By the result of the FDTD simulations, the phase of the deflected electric field is shown by the blue solid line in Fig. 3(b) as a function of $x$-coordinate at about $\mathrm{z}=3.5 \mu \mathrm{m}$. Also, the overall transmissivity through the beam deflecting metasurface is about $75 \%$ at $\mathrm{z}=3.5 \mu \mathrm{m}$.

\subsection{Design of $1 D$ flat lens}

The second Huygens' metasurface device to be demonstrated is a 1D focusing lens for the transmitted beam. To form and ideally spherical wavefront for a point-like focus at a certain focal length $f$, the phase shift distribution $\varphi(x)$ along the interface is given by

$$
\varphi(x)=\frac{2 \pi}{\lambda}\left(f-\sqrt{f^{2}+x^{2}}\right)
$$


where $\lambda$ is the vacuum wavelength of the incident light. Note that the transmission media after the lens is air. In addition, $f$ here is the predefined focal length. With the help of presented lookup data in Fig. 2(d), proper mapping can relate the required phase to the resonator radius at spatial position $x$. In the full-wave numerical FDTD simulations of the 1D lens, perfectly matched layers boundary conditions are adopted along the $x$-axis and $z$-axis and periodic boundary conditions are employed along $y$-axis. In the following designs, the focus and the aperture of the lens are chosen as $f=3.5 \mu \mathrm{m}, D=10.85 \mu \mathrm{m}$, respectively. These sizes require less computation resources and the design may be scaled to larger sizes at the same NA value of 0.85 .

In the FDTD simulation, the lens includes 17 nanodisks along the $x$-axis and has a width $D=$ $10.85 \mu \mathrm{m}$, measured from the edges of the two outermost nanodisk unit cells. The targeted phase profile of the 1D cylindrical lens from Eq. (3) is shown by blue solid line in Fig. 4(a). Theoretically available nanodisk unit cell phases at the corresponding spatial positions are also indicated by the red circles in Fig. 4(a). As also shown by the numerical results in Fig. 4(b), the full-width at half-maximum (FWHM) of the focused line for both TE and TM polarizations are $0.586 \lambda \mathrm{nm}$ for $\mathrm{NA}=0.85$ at focal length of $f=3.5 \mu \mathrm{m}$. Fig. 4(c) shows the focused intensity distribution of the transmitted field under an incident TE polarized illumination at $x z$-plane. 1D metasurface lens clearly generates a quite good focus at the designed position.

The designed 1D metasurface cylindrical lens has a transmissivity of $76 \%$. Transmission is defined as the ratio of the intensity of the transmitted light beam to that of the incident light. Note that high contrast metasurface flat lens structure shows a deviation of the focal length between the full-wave simulation analysis and the initial design, if diffraction dominates especially for small NA and small lens sizes (small Fresnel numbers) [42]. Because the designed 
lens here has high NA, geometric optics dominates and, thus, our focal length is in good agreement with the initial design.

\subsection{Design of 2 D flat lens}

The design of 2D flat lens requires additional phase gradient along the $y$-axis. Thus, the new phase distribution along the interface is given by

$$
\varphi(x, y)=\frac{2 \pi}{\lambda}\left(f-\sqrt{f^{2}+x^{2}+y^{2}}\right)
$$

Each nanodisk at position $(x, y)$ should impart the required phase given by Eq. (4). In the FDTD simulations of the 2D metasurface lens, perfectly matched layers boundary conditions are adopted along $x, y$ and $z$ directions. Fig. 5(a) illustrates the top-view of the designed 2D metasurface lens, where the aperture size is $10.85 \mu \mathrm{m} \times 10.85 \mu \mathrm{m}$. Fig. 5(b) shows the electric field intensity of the transmitted light at the focal plane. The intensity enhancement at the focal plane is achieved by more than 30 times. Due to rotational symmetry of the designed lens, focus spots for the TE and TM polarizations are exactly the same. Thus, all results are given for only TE polarization while omitting TM results. In addition, FWHM of the focused spot is $0.657 \lambda$, which is close to diffraction limited value of $0.6 \lambda$ for both TE and TM polarization as shown in Fig. 5(c). Total transmissivity of the designed 2D metasurface lens is $80 \%$ for the numerical aperture value of $\mathrm{NA}=0.85$. Depth of focus of about $1.5 \mu \mathrm{m}$ is also shown in Fig. $5(\mathrm{~d})$.

To further confirm and analyze the performance of the designed metalens, three other metalenses with different focal lengths equal to $f=6 \mu \mathrm{m}, f=10 \mu \mathrm{m}$ and $f=15 \mu \mathrm{m}$ have also been designed. The aperture size for each was taken the same as $10.85 \mu \mathrm{m}$ and, thus, all of the three metalenses are composed of $17 \times 17$ silicon nanodisks. In theory, when the focal lengths get longer, the NAs will become smaller and the intensities at the focal points will decrease accordingly. The 
intensity distributions of the transmitted light through three metalenses under the normal incidence of light are shown in Fig. 6(a), and the transmission lights are strongly and closely focused at the designed positions for all of three metalenses, respectively. For a quantitative analysis of the focusing effect of three designed metalenses, the electric field intensities along the $x$-axis and along the $z$-axis at the focal plane for the designed metalens are presented in Fig. 6(b) and Fig. 6(c), respectively. The simulated focal lengths are slightly different from the theoretical prediction, which can be mainly attributed to the diffraction effect of small size, high contrast grating lenses, as mentioned before [42]. At the focal planes, the diameters of the focal spot (FWHM along the $x$-axis) are at sub-wavelength scales, especially for smaller focal length values as shown in Fig. 6(b). The corresponding FWHMs are $1040 \mathrm{~nm}, 1140 \mathrm{~nm}$ and $1720 \mathrm{~nm}$ for three designed metalenses with focal length of $6 \mu \mathrm{m}, 10 \mu \mathrm{m}$ and $15 \mu \mathrm{m}$, respectively. As depicted in Fig. 6(c), the depths of focus (FWHM along the $z$-axis) are $4.62 \mu \mathrm{m}, 7.2 \mu \mathrm{m}$ and 16.6 $\mu \mathrm{m}$ for three designed metalenses with focal lengths of $6 \mu \mathrm{m}, 10 \mu \mathrm{m}$ and $15 \mu \mathrm{m}$, respectively. For the metalens with the shortest focal length, the shortest depth of focus and the largest transmission intensity can be achieved, as expected. Sidelobes are due the diffraction effect, which is more prominent for smaller diameter lenses with NA values smaller than 0.7 . They are suppressed especially for larger NA metalenses, as it will be illustrated by the following results.

The focusing ability of the lens is proportional to the NA which is defined as NA= $\sin \left[\tan ^{-1}(\mathrm{D} / 2 f)\right]$, where $\mathrm{D}$ is the width of the lens, and $f$ is the focal length of the lens.

For a specific focal length, as the width of the lens increases, the NA will become larger. As shown in Fig. 7(a), with plane wave illumination at normal incidence, there will be better focusing properties for the metalenses with larger NA by increasing the metalens' width. For a 
quantitative analysis of the focusing effect of three metalenses (focal length of $f=7 \mu \mathrm{m}$ ), Fig. 7(b) and Fig. 7(c) show that when NA $=0.84$, the focusing intensity increases significantly, and FWHM gets smaller compared to the metalenses with NA $=0.74$ and 0.55 . As well as the conventional lens, for the metalens with the larger NA, the size of the focal spot will be smaller, and the focusing intensity will also increase.

Various light manipulation techniques based on properly designed metasurfaces are possible including polarization independent beam focusing and steering. The targeted figure of merits such as high transmission, small FWHM at the focal point, large NA and acceptable deflecting angle are succeeded in the current study. Considering the available spectral scalability, these devices can be utilized in applications where operation at distinct known wavelengths is needed such as high power laser beam systems and fluorescence microscopy techniques. Since $1064 \mathrm{~nm}$ wavelength is of great importance for high power applications and second harmonic generation applications, proposed design focused on that part of the spectrum in the present study.

The proposed metasurface flat lens can be realized using a silicon-on-insulator (SOI) wafer with a buried oxide (BOX) of thickness $350 \mathrm{~nm}$ and top Si layer thickness of $170 \mathrm{~nm}$. The wafers are spin-coated by a negative-tone electron beam resist and electron beam lithography (EBL) is used to define the desired 2D patterns, providing extraordinary in-plane design freedom and precision control. After exposure and development, the resulting resist pattern is directly employed as an etch mask in a reactive ion etching of the silicon thin film and residual resist can be removed by using oxygen plasma. A plasma enhanced chemical vapor deposition (PECVD) $\mathrm{SiO}_{2}$ is then deposited covering the etched Si cylinders, to achieve the desired substrate thickness of $700 \mathrm{~nm}$. 
After patterning the back side oxide of the SOI wafer, a Tetramethylammonium Hydroxide (TMAH) etching should be used to remove the bulk Si below the lens [30].

\section{Conclusion}

In conclusion, all-dielectric Huygens' metasurfaces capable of serving as polarization insensitive beam deflection element and lens for the transmitted beam using high contrast, subwavelength silicon nanodisks are proposed. By rigorous numerical simulations, optimum design parameter region with high transmission and sufficient phase control was achieved. In addition, flat metasurface lens with high numerical aperture value of 0.85 and with transmissivity as high as $80 \%$ and a metasurface beam deflection device with beam deflecting angle of $6.9^{\circ}$ with transmissivity of $75 \%$ have been obtained. The design is very compact, thin and offers less alignment problem comparing to bulky conventional lenses composed of many surfaces. Since the proposed system can easily handle complex wavefront manipulation, it has a great promise for other beam shaping applications. By playing with the asymmetry of the unit cells used in the design, the system can also be made polarization sensitive. Drawbacks of plasmonic metasurfaces using complex split-ring resonator design with intrinsic Ohmic loss are overcome by using all-dielectric silicon nanodisks as the basic meta-atoms. The design is scalable to other wavelengths and is also easy to fabricate with standard single step electron beam lithography based conventional planar semiconductor fabrication methods due to very small aspect ratio of the designed structures. Therefore, there is a great application prospect in the field of integrated optics via utilizing metasurfaces in the design. 


\section{AUTHOR INFORMATION}

Corresponding Authors

E-mail: aozdemir@optics.arizona.edu.

The authors declare no competing financial interest.

\section{Acknowledgement}

H.K. gratefully acknowledges the partial support of the Turkish Academy of Sciences.

\section{References}

[1] N. Engheta, R.W. Ziolkowski, Metamaterials: Physics and Engineering Explorations, 2006. doi:10.1002/0471784192.

[2] N.I. Zheludev, Y.S. Kivshar, From metamaterials to metadevices, Nat. Mater. 11 (2012) 917-924. doi:10.1038/nmat3431.

[3] N. Yu, F. Capasso, Flat optics with designer metasurfaces, Nat. Mater. 13 (2014) 139150. doi:10.1038/nmat3839.

[4] A. V. Kildishev, A. Boltasseva, V.M. Shalaev, Planar Photonics with Metasurfaces, Science 339 (2013) 1289. doi:10.1126/science.1232009.

[5] N. Meinzer, W.L. Barnes, I.R. Hooper, Plasmonic meta-atoms and metasurfaces, Nat. Photonics. 8 (2014) 889-898. doi:10.1038/nphoton.2014.247.

[6] M. Khorasaninejad, F. Aieta, P. Kanhaiya, M.A. Kats, P. Genevet, D. Rousso, F. Capasso, Achromatic metasurface lens at telecommunication wavelengths, Nano Lett. 15 (2015) 5358-5362. doi:10.1021/acs.nanolett.5b01727.

[7] M. Khorasaninejad, A.Y. Zhu, C. Roques-Carmes, W.T. Chen, J. Oh, I. Mishra, R.C. Devlin, F. Capasso, Polarization-insensitive metalenses at visible wavelengths, Nano Lett. 16 (2016) 7229-7234. doi:10.1021/acs.nanolett.6b03626.

[8] F.C. F.Aieta, P.Genevet, M.A.Kats, N.Yu, R.Blanchard, Z.Gaburro, Aberration-free ultrathin flat lenses and axicons at telecom wavelengths based on plasmonic metasurfaces, Nano Lett. 12 (2012) 4932- 4936.

[9] M. Khorasaninejad, W.T. Chen, A.Y. Zhu, J. Oh, R.C. Devlin, C. Roques-Carmes, I. Mishra, F. Capasso, Visible wavelength planar metalenses based on titanium dioxide, IEEE J. Sel. Top. Quantum Electron. (2016) 1-16. doi:10.1109/JSTQE.2016.2616447.

[10] M. Khorasaninejad, W.T. Chen, R.C. Devlin, J. Oh, A.Y. Zhu, F. Capasso, Metalenses at visible wavelengths: Diffraction-limited focusing and subwavelength resolution imaging, Science 352 (2016) 1190-1194. doi:10.1126/science.aaf6644.

[11] A. Arbabi, Y. Horie, A.J. Ball, M. Bagheri, A. Faraon, Subwavelength-thick lenses with high numerical apertures and large efficiency based on high contrast transmitarrays, Nat. Commun. 6 (2015) 1-10. doi:10.1038/ncomms8069. 
[12] A. Arbabi, R.M. Briggs, Y. Horie, M. Bagheri, A. Faraon, Efficient dielectric metasurface collimating lenses for mid-infrared quantum cascade lasers, Opt. Express. 23 (2015) 33310-33317. doi:10.1364/OE.23.033310.

[13] B. Memarzadeh, H. Mosallaei, Array of planar plasmonic scatterers functioning as light concentrator, Opt. Lett. 36 (2011) 2569-2571. doi:10.1364/OL.36.002569.

[14] B. Zhang, J. Hendrickson, N. Nader, H. Chen, J. Guo, Metasurface optical antireflection coating metasurface optical antireflection coating, Appl. Phys. Lett. 105 (2014) 241113. doi:10.1063/1.4904827.

[15] D. Lin, P. Fan, E. Hasman, M.L. Brongersma, Dielectric gradient metasurface optical elements, Science 345 (2014) 5189-5192.

[16] N.K. Grady, J.E. Heyes, D.R. Chowdhury, Y. Zeng, M.T. Reiten, A.K. Azad, A.J. Taylor, D. A. R. Dalvit, H.-T. Chen, Terahertz metamaterials for linear polarization conversion and anomalous refraction., Science. 340 (2013) 1304-1307. doi:10.1126/science.1235399.

[17] X.-L. Cai, J.-W. (University of B. Wang, M.J. Strain, B. Johnson-Morris, J.-B. Zhu, M. Sorel, J.L. O'Brien, M.G. Thompson, S.-Y. Yu, Integrated compact optical vortex beam emitters, Science. 338 (2012) 363-366. doi:10.1126/science.1226528.

[18] K. Aydin, V.E. Ferry, R.M. Briggs, H.A. Atwater, Broadband polarization-independent resonant light absorption using ultrathin plasmonic super absorbers, Nat. Commun. 2 (2011) 517. doi:10.1038/ncomms 1528.

[19] Y. Ke, Y. Liu, Y. He, J. Zhou, H. Luo, S. Wen, Realization of spin-dependent splitting with arbitrary intensity patterns based on all-dielectric metasurfaces, Appl. Phys. Lett. 107 (2015) 041107. doi:10.1063/1.4927479.

[20] Y. Ke, Y. Liu, J. Zhou, Y. Liu, H. Luo, S. Wen, Photonic spin filter with dielectric metasurfaces, Opt. Express. 23 (2015) 33079-33086. doi:10.1364/OE.23.033079.

[21] Y. Ke, Y. Liu, J. Zhou, Y. Liu, H. Luo, S. Wen, Optical integration of PancharatnamBerry phase lens and dynamical phase lens, Appl. Phys. Lett. 108 (2016) 101102. doi:10.1063/1.4943403.

[22] Y. Ke, Z. Liu, Y. Liu, J. Zhou, W. Shu, H. Luo, S. Wen, Compact photonic spin filters, Appl. Phys. Lett. 109 (2016) 181104. doi:10.1063/1.4966954.

[23] P.R. West, S. Ishii, G. V. Naik, N.K. Emani, V.M. Shalaev, A. Boltasseva, Searching for better plasmonic materials, Laser Photonics Rev. 4 (2010) 795-808.

doi:10.1002/lpor.200900055.

[24] A. Boltasseva, H.A. Atwater, Low-loss plasmonic metamaterials, Science. 331 (2011) 290-291. doi:10.1126/science.1198258.

[25] L. Huang, X. Chen, B. Bai, Q. Tan, G. Jin, T. Zentgraf, S. Zhang, Helicity dependent directional surface plasmon polariton excitation using a metasurface with interfacial phase discontinuity, Light Sci. Appl. 2 (2013) e70. doi:10.1038/lsa.2013.26.

[26] W. Wang, Z. Guo, R. Li, J. Zhang, Y. Li, Y. Liu, X. Wang, S. Qu, Plasmonics metalens independent from the incident polarizations, Opt. Express. 23 (2015) 16782-16791. doi:10.1364/OE.23.016782.

[27] H. Shao, J. Wang, D. Liu, Z. Da Hu, X. Xia, T. Sang, Plasmonic planar lens based on slanted nanoslit array, Plasmonics 12 (2016) 1-7. doi:10.1007/s11468-016-0272-8.

[28] J.V.D. Groep, A. Polman, Designing dielectric resonators on substrates: Combining magnetic and electric resonances, Opt. Express. 21 (2013) 1253-1257. doi:10.1364/OE.21.026285.

[29] C. Pfeiffer, A. Grbic, Metamaterial Huygens' surfaces: tailoring wave fronts with 
reflectionless sheets, Phys. Rev. Lett. 110 (2013) 197401.

[30] M. Decker, I. Staude, M. Falkner, J. Dominguez, D.N. Neshev, I. Brener, T. Pertsch, Y.S. Kivshar, High-efficiency dielectric huygens' surfaces, Adv. Opt. Mater. 3 (2015) 813820. doi:10.1002/adom.201400584.

[31] I. Staude, A.E. Miroshnichenko, M. Decker, N.T. Fofang, S. Liu, E. Gonzales, J. Dominguez, T.S. Luk, D.N. Neshev, I. Brener, Y. Kivshar, Tailoring directional scattering through magnetic and electric resonances in subwavelength silicon nanodisks, ACS Nano. 7 (2013) 7824-7832. doi:10.1021/nn402736f.

[32] Y.F. Yu, A.Y. Zhu, R. Paniagua-Domínguez, Y.H. Fu, B. Luk'yanchuk, A.I. Kuznetsov, High-transmission dielectric metasurface with $2 \pi$ phase control at visible wavelengths, Laser Photonics Rev. 9 (2015) 412-418. doi:10.1002/lpor.201500041.

[33] K.E. Chong, L. Wang, I. Staude, A.R. James, J. Dominguez, S. Liu, G.S. Subramania, M. Decker, D.N. Neshev, I. Brener, Y.S. Kivshar, Efficient polarization-insensitive complex wavefront control using Huygens' metasurfaces based on dielectric resonant meta-atoms, ACS Photonics. 3 (2016) 514-519. doi:10.1021/acsphotonics.5b00678.

[34] K.E. Chong, I. Staude, A. James, J. Dominguez, S. Liu, S. Campione, G.S. Subramania, T.S. Luk, M. Decker, D.N. Neshev, I. Brener, Y.S. Kivshar, Polarization-independent silicon metadevices for efficient optical wavefront control, Nano Lett. 15 (2015) 53695374. doi:10.1021/acs.nanolett.5b01752.

[35] M. Kerker, D.-S. Wang, C.L. Giles, Electromagnetic scattering by magnetic spheres, J. Opt. Soc. Am. 73 (1983) 765. doi:10.1364/JOSA.73.000765.

[36] X. Wang, C. Chen, L. Pan, J. Wang, A graphene-based Fabry-Pérot spectrometer in midinfrared region, Sci. Rep. 6 (2016) 32616. doi:10.1038/srep32616.

[37] Y. Shen, X. Luo, Efficient bending and focusing of light beam with all-dielectric subwavelength structures, Opt. Commun. 366 (2016) 174-178. doi:10.1016/j.optcom.2015.12.043.

[38] E. Palik, Handbook of Optical Constants of Solids, 1997. doi:10.1016/B978-0125444156.50143-6.

[39] F. Silvestri, G. Gerini, S. Mbbäumer, E.J.V. Zwet, Robust design procedure for dielectric resonator metasurface lens array, Opt. Express 24 (2016) 29153-29169.

[40] V. Egorov, M. Eitan, J. Scheuer, Genetically optimized all-dielectric metasurfaces, Opt. Express 25 (2017) 2583-2593. doi:10.1364/OE.25.002583.

[41] N. Yu, P. Genevet, M. a Kats, F. Aieta, J.-P. Tetienne, F. Capasso, Z. Gaburro, Light propagation with phase discontinuities reflection and refraction, Science 334 (2011) 333337. doi:10.1126/science.1210713.

[42] S. He, Z. Wang, Q. Liu, W. Wang, Study of focal shift effect in planar GaN high contrast grating lenses, Opt. Express 23 (2015) 2-6. doi:10.1364/OE.23.029360. 
a

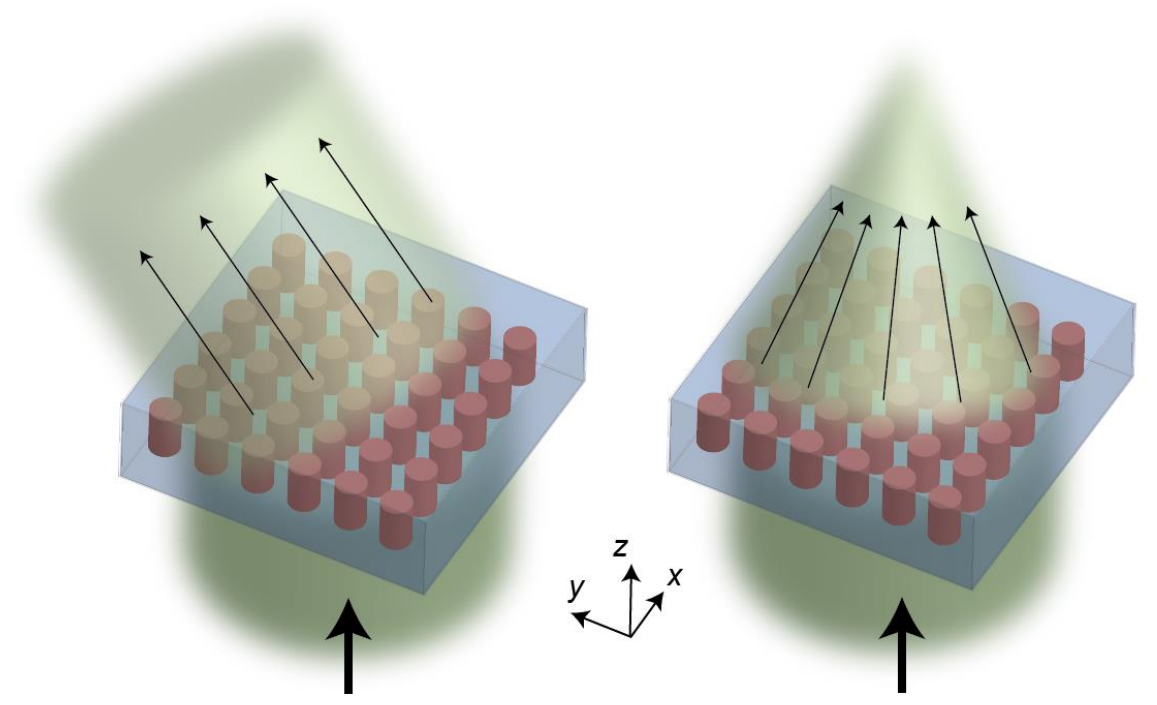

b

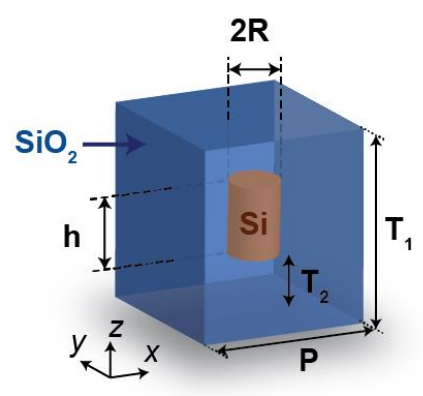

Fig. 1. (a) Artistic impression of an all-dielectric resonator metasurface beam deflector and lens (b) Side-view of the unit cell of the designed metasurface lens: a Silicon nanodisk embedded in a host substrate of fused Silica $\left(\mathrm{SiO}_{2}\right)$. Here nanodisks are arranged in a square lattice array. For the design wavelength $\lambda=1064 \mathrm{~nm}$, the unit cell dimension is equal to $\mathrm{P}=620 \mathrm{~nm}$, nanodisk height is equal to $\mathrm{H}=170 \mathrm{~nm}$, and the nanodisk radii $(\mathrm{R})$ vary between 130 to $240 \mathrm{~nm}$. The host Silica thickness values are equal to $T_{1}=700 \mathrm{~nm}, \mathrm{~T}_{2}=350 \mathrm{~nm}$. The $500 \mu \mathrm{m}$ thick back layer of fused Silica is not shown in for the sake of clarity. 

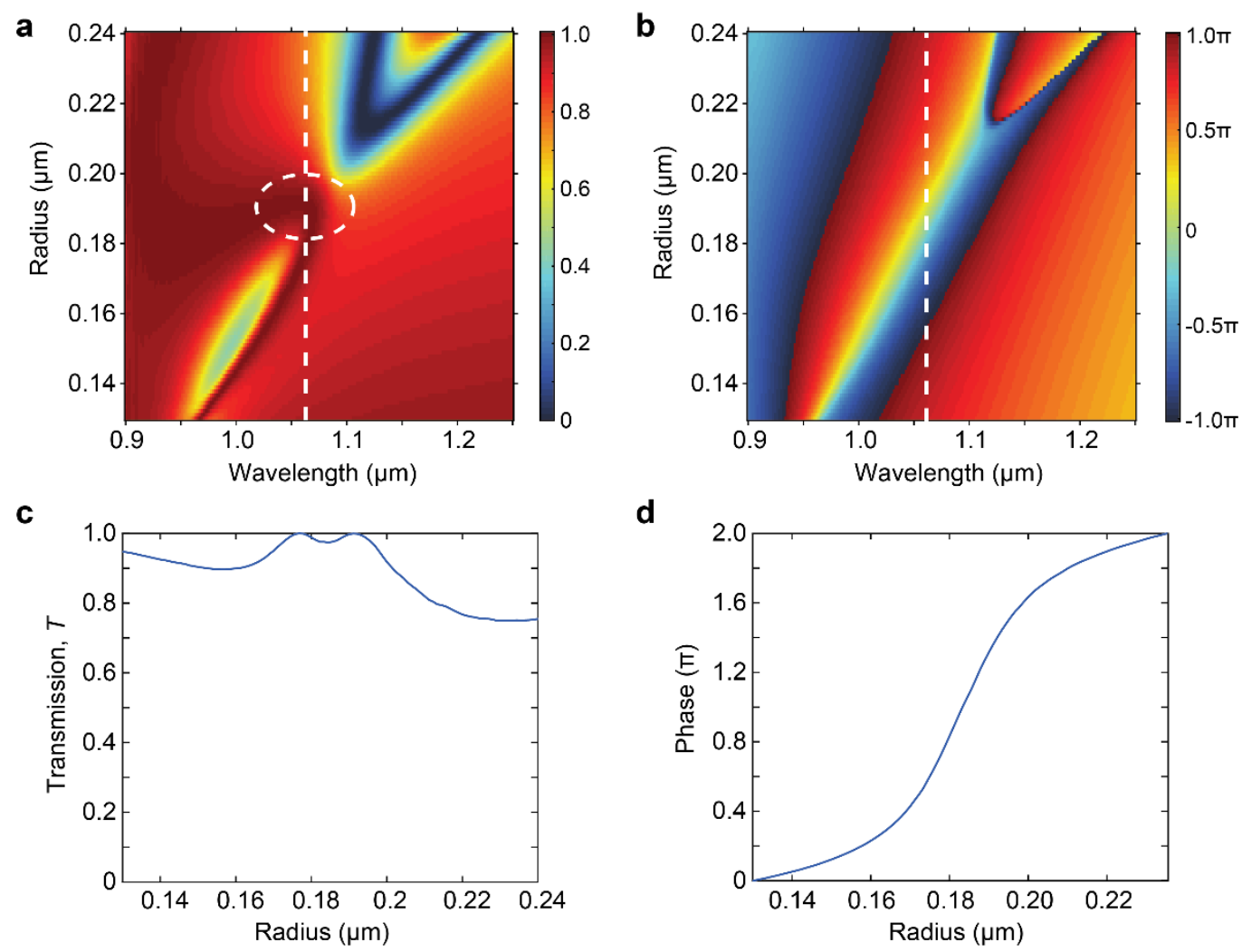

Fig. 2. (a) Transmission and (b) Phase modulation of nanodisk arrays versus wavelength and nanodisk radii with nanodisk height of $170 \mathrm{~nm}$ and $620 \mathrm{~nm}$ unit cell dimension. Electric dipole (ED) and magnetic dipole (MD) resonance overlap occurs at $1064 \mathrm{~nm}$ shown by the vertical line, and overlap region is shown by the white ellipse. (c) Transmission and (d) phase shift at a wavelength of $1064 \mathrm{~nm}$ for varying radii of nanodisks calculated from numerical simulations. 

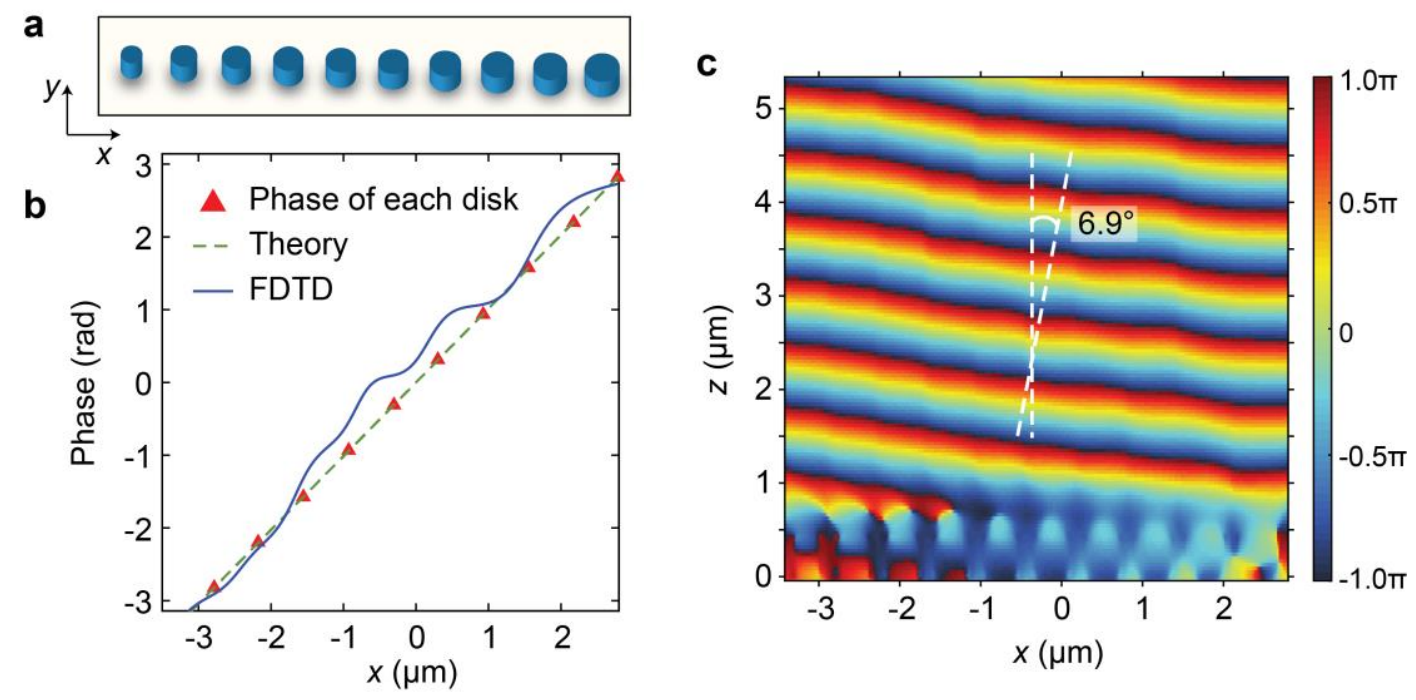

Fig. 3. (a) Super cell with 10 nanodisks in a periodic array of the beam deflector. It repeats with a period of $6.1 \mu \mathrm{m}$ along the $x$-axis and $620 \mathrm{~nm}$ along the $y$-axis. (b) Phase of the electric field (blue line) as a function of $x$-coordinate at about $z=3.5 \mu \mathrm{m}$. The dashed green line and red triangles show the targeted phase profile and phase of each nanodisk, respectively. (c) Phase of the transmitted field $E_{x}$ when the plane wave passes through the dielectric metasurface beam deflector. 

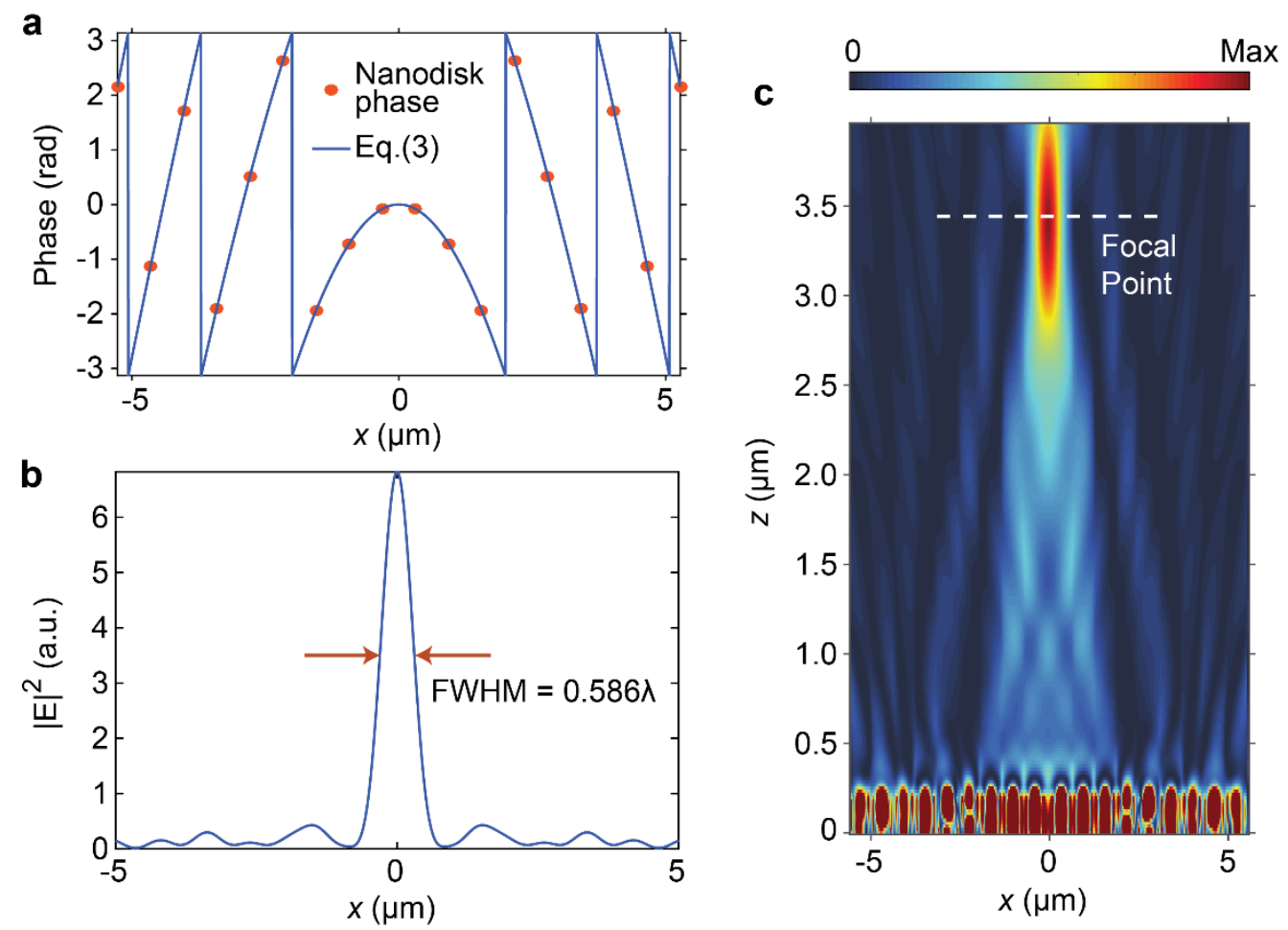

Fig. 4. Typical focusing performance of the $1 \mathrm{D}$ cylindrical metasurface lens. (a) The targeted phase profile (blue solid line) and phase of each nanodisk unit cells (red circles) of the designed lens with a focal length of $3.5 \mu \mathrm{m}$ are also shown. (b) Electric field intensity profile in the focal plane $(z=3.5 \mu \mathrm{m}, \mathrm{y}=0)$. (c) Electric field intensity distribution of the transmitted field at wavelength of $1064 \mathrm{~nm}$. 


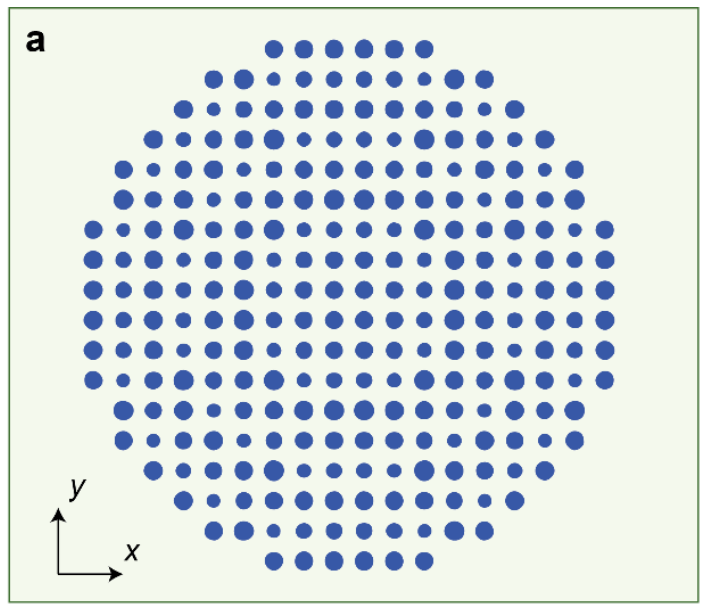

b
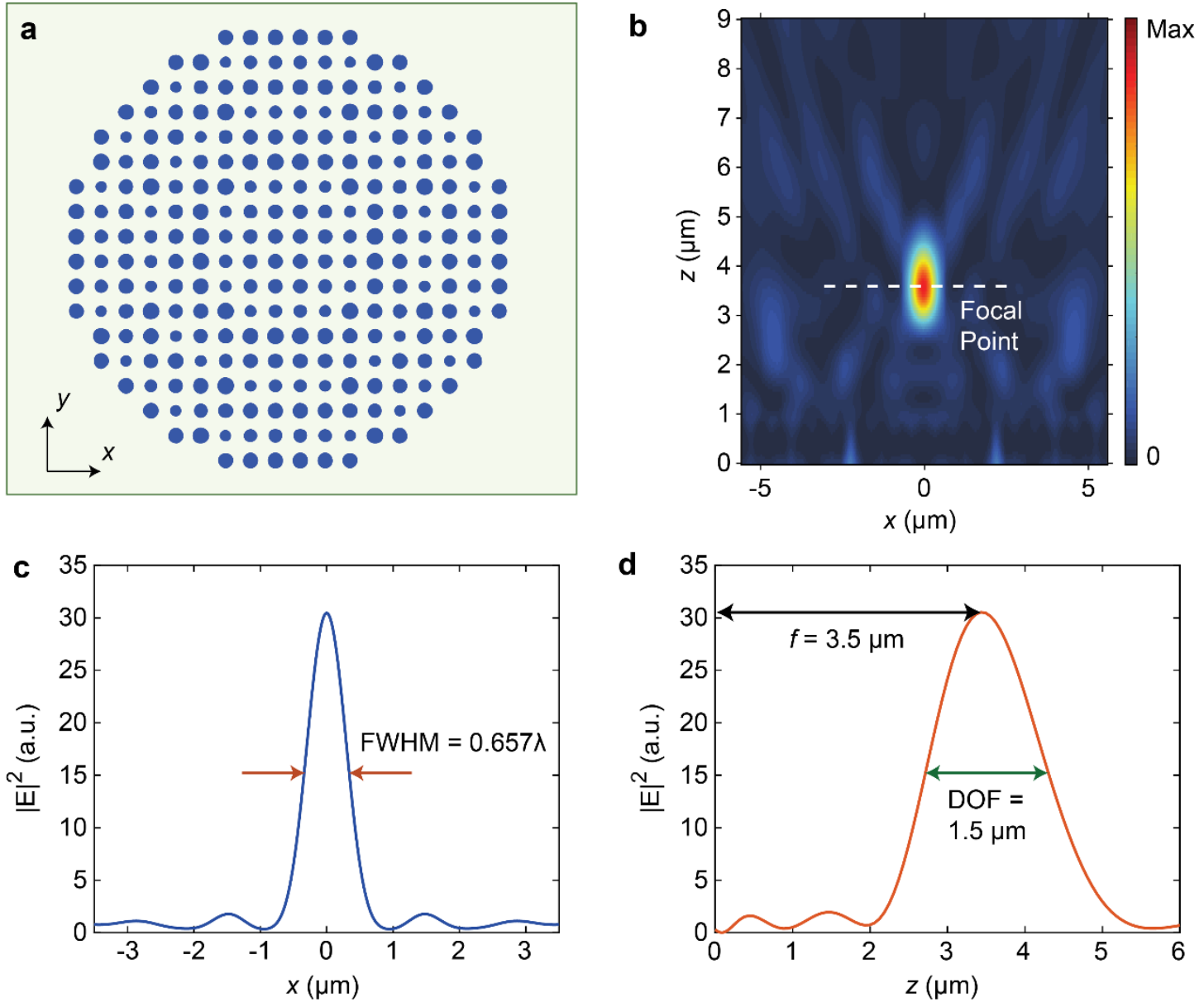

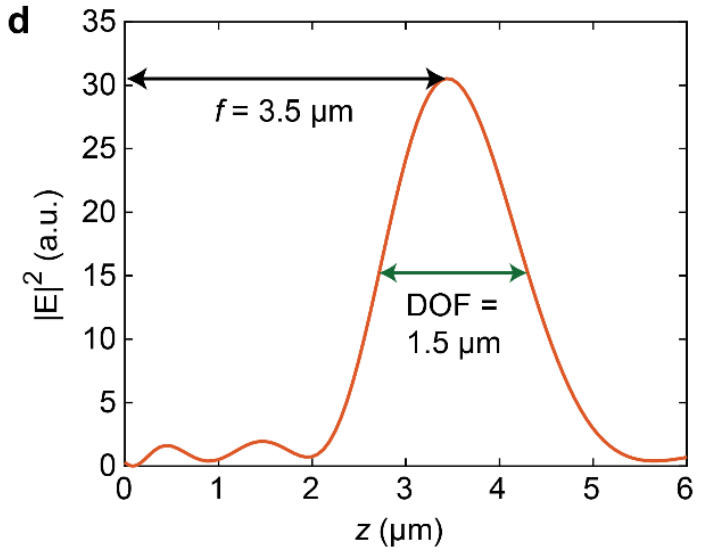

Fig. 5. (a) Top-view of the designed all-dielectric flat lens (b) Distribution of the electric field intensity in the $x z$ plane, (c) and (d) are the field intensity distribution along $x$-direction (at $z=f, y=0$ ) and $z$-direction, respectively. The FWHM of the spot is $0.657 \lambda$, focal length is $f=3.5 \mu \mathrm{m}$ and Depth of focus is about $1.5 \mu \mathrm{m}$. 

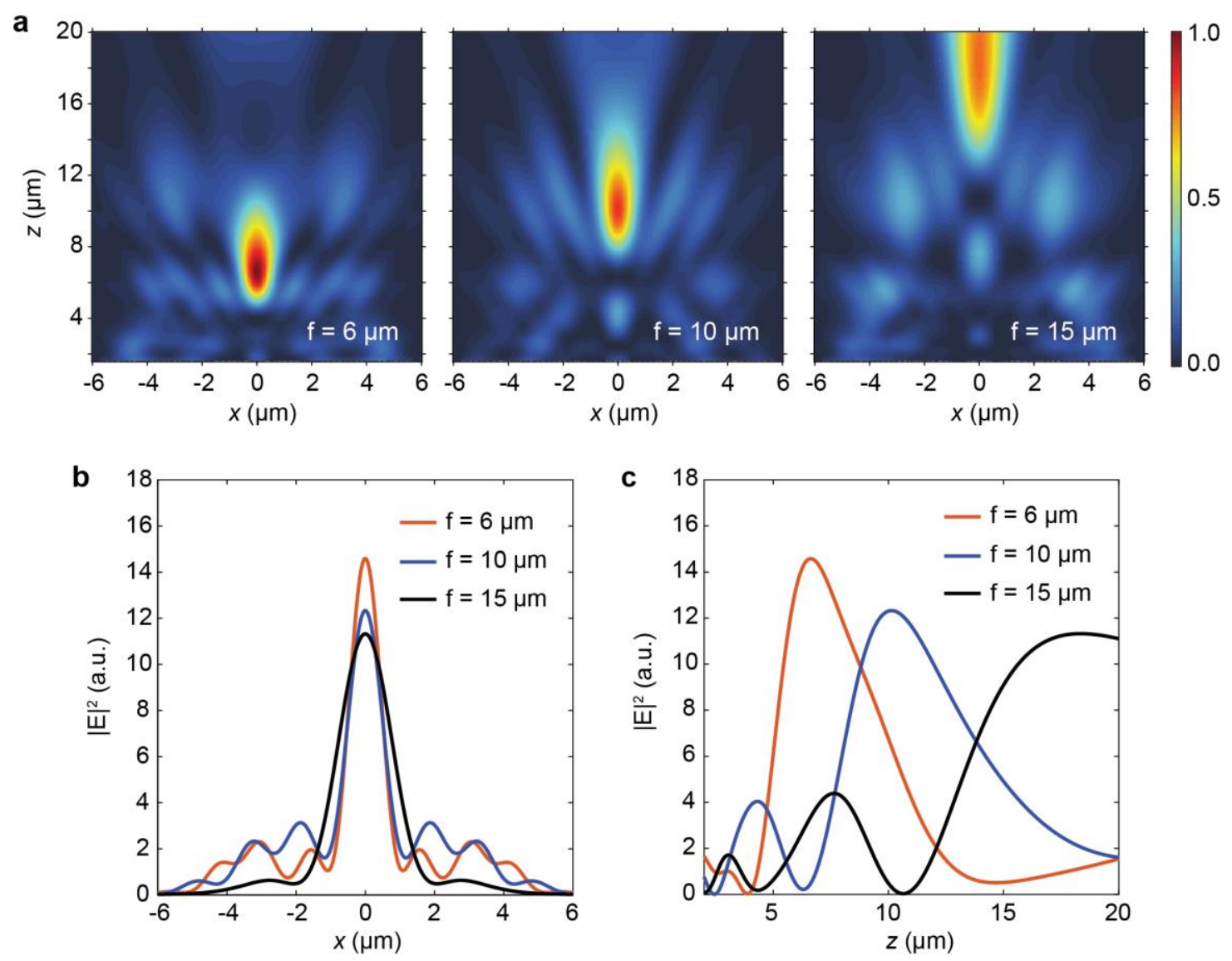

Fig. 6. (a) Intensity distributions of the transmitted light in the $x z$-plane, through the designed metalenses with different focal lengths of $6 \mu \mathrm{m}, 10 \mu \mathrm{m}, 15 \mu \mathrm{m}$, respectively, under the normally incident light with a wavelength of $1064 \mathrm{~nm}$. Diameter of metalens for all is $10.85 \mu \mathrm{m}$ (b) The electric field intensity along the $x$-axis and (c) the electric field intensity along the $z$-axis at the corresponding focusing planes with different focal lengths. 

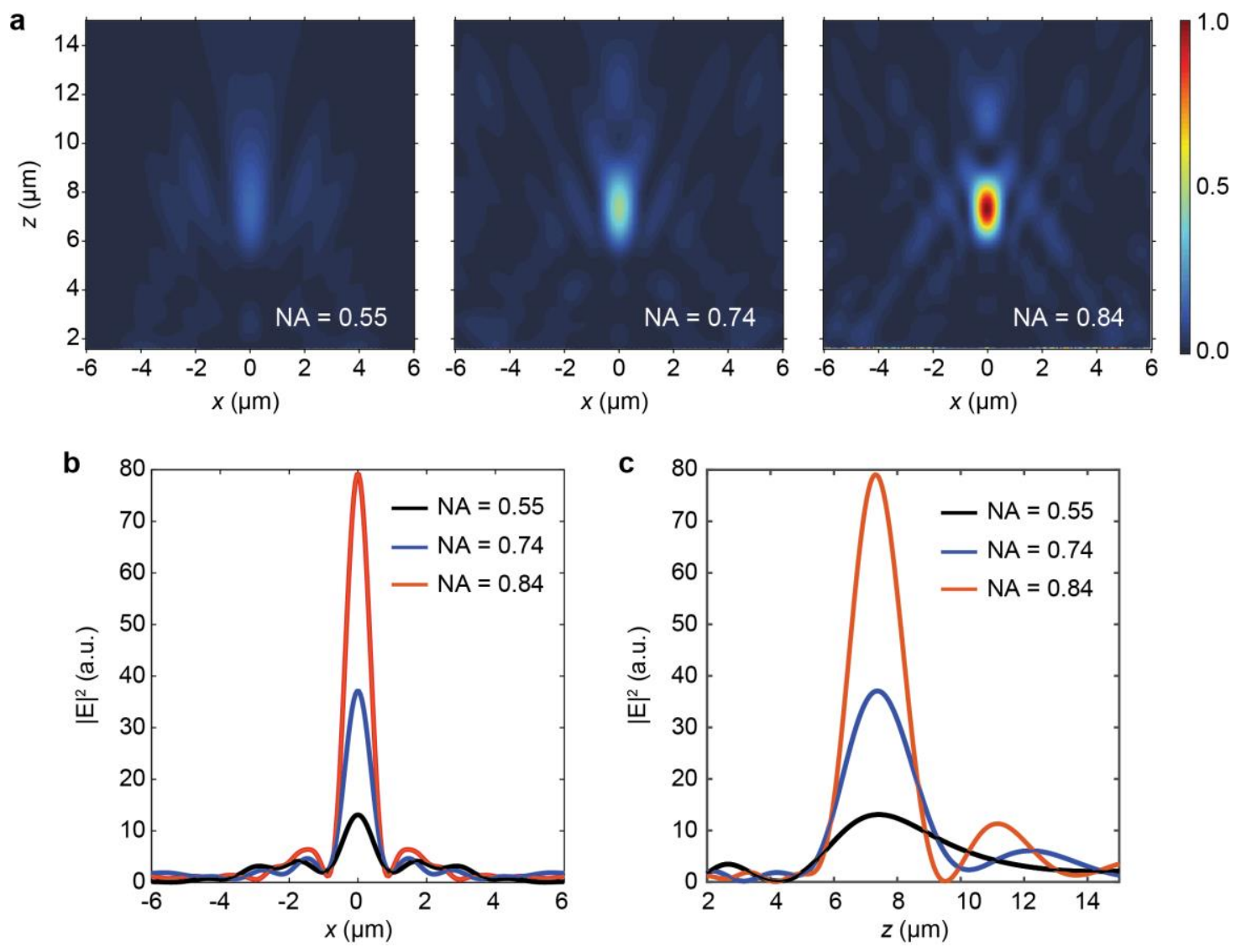

Fig. 7. (a) Intensity distributions of the transmitted light in the $x z$-plane, through the designed metalenses with different NA values of $0.55,0.74,0.84$, respectively, under the normally incident light with a wavelength of 1064 $\mathrm{nm}$. The designed focal length for all is $7 \mu \mathrm{m}$, and the diameter of metalenses for each is $9.3 \mu \mathrm{m}, 15.55 \mu \mathrm{m}, 21.07$ $\mu \mathrm{m}$, respectively. (b) The electric field intensity along the $x$-axis and (c) the electric field intensity along the $z$-axis at the corresponding focusing planes with different NA values. 